hormonal treatment of endometriosis may be the recently introduced luteinizing hormone-releasing hormone agonist buserelin. Both drugs are known to be effective in reducing endometriotic implants.

\title{
A Comparison of the Changes in the Plasma Lipids and Lipoproteins during Therapy with Danazol and Gestrinone for Endometriosis
}

\author{
M.W. Booker“, P.R. Turnerb, B. Lewisb, M.I. Whitehead“ \\ "Academic Department of Obstetrics and Gynaecology, \\ King's College School of Medicine and Dentistry, Denmark Hill, London; \\ b Department of Chemical Pathology and Metabolic Diseases, \\ St Thomas' Hospital Medical School, London, England
}

Danazol and gestrinone are effective drugs for treating endometriosis. Danazol has an adverse, but reversable effect on the plasma lipids and lipoproteins. The effect of gestrinone has not previously been studied.

Twenty endometriosis patients were randomly allocated to 6 months therapy with either danazol $200 \mathrm{mg} /$ day or gestrinone $2.5 \mathrm{mg}$ twice weekly. Fasting blood samples were taken before, during and after treatment for a full lipid analysis by ultracentrifugation. There were no significant changes in total cholesterol, total triglycerides, very lowdensity lipoprotein (VLDL) cholesterol, VLDL triglycerides, or LDL cholesterol. Both drugs produced a significant fall in the levels of high-density lipoprotein (HDL) cholesterol, HDL2 cholesterol and HDL3 cholesterol. There were significant differences between the two treatments and the levels returned to normal on stopping treatment. Although a temporary lowering of the level of HDL cholesterol is an adverse effect, it is probably accetable in view of the clinical benefits of treatment.

Endocrine and Morphological Changes during GnRH

Analogue Treatment (Suprefact) for Endometriosis

C. MelegaM. FilicoriA. PareschiG. Possati C. Flamigni1', S. Barnabeb "Department of Gynecologic Endocrinology of Bologna University (Prof. C. Flamigni); bllnd Obstetric and Gynecological Clinic (Prof. C. Orlandi), Bologna, Italy

A potent agonist of gonadotropin-releasing hormone ( $\mathrm{GnRH}$; Suprefact-Hoechst) was administered to 5 patients affected by endometriosis diagnosed by laparoscopy. Classification of endometriosis was made, according to the American Fertility Society, at the time of laparoscopy. Suprefact-Hoechst was adminstered subcutaneously $(0.3$ 
mg twice a day) for 6 months. 